\section{GW23-e1104 THE REASEARCH INTO THE CLINICAL CHARACTERISTICS OF PERIPARTUM CARDIOMYOPATHY}

doi:10.1136/heartjnl-2012-302920j.17

Fang Zhang, Xuebin. Geriatric Cardiovascular Disease Center of Chinese PLA, Department of Cardiology, No.252 Hospital of PLA, Baoding, Hebei

Objectives The research is to find the related risk factors that may lead to PPCM through analysing and summarising the general clinical characteristics of PPCM patients. NT-proBNP is used to diagnose the heart failure; cInI is the preferred biochemical index of myocardial injury and D-dimer is used to diagnose the thrombosis embolism and DIC. The research investigated the clinical values of the above three factors in PPCM's early diagnosis, differential diagnosis, treatment and prognostic evaluations.

Methods In this research, 79 patients classified as PPCM, 30 patients classified as late pregnancy physiological responses and 35 patients classified as healthy peripartum are selected. Firstly, the clinical data of 79 cases of patients was collected and the test results of NT-proBNP, cInI and D-dimer in the clinical documents were analysed from two angles of view:hemodynamic and myocardial cell damage. The NT-proBNP levels of sample were tested by domestic ssg-2 multi-function immune detector and the reagents were golden standard card. The measured values exceeding the mean value of healthy peripartum of 2 times were regarded as positive. The test of cInI was done by American Boshi Triage analyser through fluorescence immunoassay method. The reference value was less than $0.4 \mathrm{ng}$ per $\mathrm{ml}$. The test of $\mathrm{D}$-dimer was done by Japan made sysmex CA7000 analyser through immune turbidimetry method. The reference value was between zero and 0.55 per Liter. The clinical significance of the three indexes in three classified patients were carefully observed.

\section{Results}

1. The PPCM in this region the total incidence rate was $6.90 \%$, the last 3 months of pregnancy incidence was $24.05 \%$, within 3 months postpartum onset was $67.09 \%$, postpartum 3 months after the onset of $8.86 \%$, urban area accounts for $7.59 \%$, the county accounted for $20.25 \%$, accounted for $72.15 \%$ of the rural cadres, workers accounted for $6.33 \%, 15.19 \%$, the farmer occupies $78.48 \%$, advanced maternal age accounted for $50.63 \%$, prolific accounted for 81.01, anaemia, hypertension accounted for $44.30 \%$, accounted for $41.77 \%$, malnutrition accounted for $89.87 \%$

2. In PPCM major clinical manifestations of left heart insufficiency and early HF diagnosis of left ventricular systolic performance, similar to HF, severe right heart failure and whole heart failure occurs when the performance of routine laboratory tests; most of the inflammatory response, aneamia, low protein, endocrine disorders, and abnormal coagulation performance; $\mathrm{X}$ line inspection mainly for the left cardiac insufficiency cardiac left enlargement, or a generally increased, and pericardial effusion is not easy to distinguish; ECG examination showed left ventricular hypertrophy and strain change and all kinds of arrhythmia; UCG examination showed left ventricular systolic function, EF and FS were decreased.

3. In 19 cases of PPCM in the last 3 months of pregnancy in 94.74\% patients undergoing caesarean section.

4. Compared with healthy contrast group, the Logarithm of serum NT-proBNP of PPCM group and physiological responses group during trimester of pregnancy rise significantly. The serum $\mathrm{cTnI}$ of PPCM group are higher significantly than healthy contrast group but the serum cTnI of physiological responses group during trimester of pregnancy has no statistical significance compared with healthy perinatal contrast group $(p>0.05)$. Because of Physiological cardiac enlargement or heart shift due to Diaphragm elevation in Physiological response group, the Ventricular volume overload changed but there are no HF and myocardial injury. Due to HF that increased the overload of ventricular volume and myocardial injury, the level of NT-proBNP and cTnI both rise in PPCM group. (5) Compared with healthy contrast group, the Logarithm of serum NT-proBNP of PPCM group and physiological responses group during trimester of pregnancy raised significantly. The serum cTnI of PPCM group were higher significantly than healthy control group but the serum cTnI of physiological responses group during trimester of pregnancy had no statistical significance compared with healthy perinatal contrast group $(p>0.05)$. Because of physiological cardiac enlargement or heart shift due to Diaphragm elevation in physiological response group, the ventricular volume overload changed but there were no HF or myocardial injury. Due to HF that increased the overload of ventricular volume and myocardial injury, the level of NT-proBNP and cTnI both rise in PPCM group.

5. Compared with those of healthy perinatal control group, serum cTnI levels of PPCM of the early stage NYHA I subgroup rise significantly, while NT-proBNP difference had no statistical significance. NT-proBNP levels rise significantly in physiological responses group and the difference of serum $\mathrm{cTnI}$ had no statistical significance. Compared with healthy control group, the above two indexes rise significantly in PPCM early stage NYHA II subgroup and the difference had statistical significance. Because the patients of PPCM early stage NYHA I level had gotten myocardial injury but there was no HF, cTnI rise significantly. PPCM early stage patients had gotten I level HF and myocardial injury at the same time. Although physiological response group had no HF and myocardial injury, ventricular volume overload changed due to cardiac enlargement or shift that led to the values of serum cTnI changed not obviously but the logarithm of NT-proBNP rise significantly.

6. The sensitivity of serum NT-proBNP in early stage diagnosis of PPCM was relatively high. Especially for PPCM early stage NYHA II level diagnosis, the sensitivity was 100 percent but specificity and negative forecast number was relatively low and positive forecast number was less than 55 percent. The sensitivity, specificity, negative and positive forecast number of serum cTnI for PPCM early stage diagnosis were higher than 90 percent.

7. Compared with those of healthy control group, D-dimer level of physiological responses group rise significantly. Compared with those of physiological response group, D-dimer level PPCM group rise significantly. The length of stay in hospital were 


\section{ABSTRACTS}

prolonged in those whose D-dimer rise high in PPCM group.

Conclusions PPCM in this region the total incidence rate was $6.90 \%$, with 3 months postpartum onset to see more, rural city, farmers, workers and cadres, prolonged malnutrition, endocrine disorders, prenatal care, maternal age, less prolific, caesarean section, hypertension, inflammation, and closely related to the onset of PPCM. PPCM in patients with caesarean section rate is far greater than the rate of vaginal delivery; clinical manifestations of PPCM and related auxiliary examination for left ventricular dysfunction and early performance of HF; combined test of two indexes of NTproBNP and cTnI is helpful of for PPCM early diagnosis and differential diagnosis. The differential diagnosis value of serum cTnI for PPCM early stage diagnosis is higher than that of NT-proBNP. The D-dimer level of PPCM patients rise significantly and the length of stay in hospital for these patients were prolonged significantly compared with that of those whose $\mathrm{D}$-dimer is normal. 